\title{
Transición a la maternidad/ paternidad y prácticas de crianza en tres generaciones
}

Transition to maternity/paternity and rearing practices in three generations

Transição para maternidade / paternidade e práticas de criação em três gerações

\author{
Erika Robles-Estrada ${ }^{1}$ \\ Hans Oudhof-van Barneveld ${ }^{2}$ \\ Aída Mercado-Maya ${ }^{3}$
}

Recibido: 11 de marzo de 2019

Aprobado: 14 de noviembre de 2019

Publicado: 20 de noviembre de 2019

Cómo citar este artículo:

Robles-Estrada E., Oudhof-van Barnev H. y Mercado-Maya, A. (2019). Transición a la maternidad/paternidad y prácticas de crianza en tres generaciones.

Pensando Psicología, 15(26), 1-28.

DOI: https://doi.org/10.16925/2382-3984.2019.02.02

Artículo de investigación. https://doi.org/10.16925/2382-3984.2019.02.02

1 Universidad Autónoma del Estado de México

ORCID: https://orcid.org/0000-0001-7438-4500

Correo electrónico: nberriogarc@uniminuto.edu.co

2 Universidad Autónoma del Estado de México

ORCID: https://orcid.org/0000-0003-1265-3052

3 Universidad Autónoma del Estado de México

ORCID: https://orcid.org/0000-0003-0672-9524 


\section{Resumen}

Objetivo: se analizó la transición a la maternidad/paternidad y la percepción de las prácticas de crianza en tres generaciones de 18 hombres y mujeres de la ciudad de Toluca, México.

Metodología: se empleó la entrevista a profundidad y para el análisis de la información, el método fenomenológico.

Resultados: la transición a la maternidad/paternidad mantuvo elementos constantes entre generaciones, así como en la práctica de crianza del fomento de las buenas relaciones entre hermanos. Los aspectos dinámicos estuvieron en el cuidado de la salud física y mental, control coercitivo y apoyo escolar. La práctica constante en las dos primeras generaciones y dinámica en la tercera fue, enseñar el papel de niña o niño.

Conclusiones: se concluye que la transición a la maternidad/paternidad es un proceso interno, mientras que las prácticas de crianza dependen del contexto sociocultural.

Palabras claves: maternidad, paternidad, prácticas de crianza.

\section{Abstract}

Objective: To analyze the transition to maternity / paternity and the perception of parenting practices in three generations of 18 men and women from the city of Toluca, Mexico.

Methodology: In-depth interviews were performed and the phenomenological method was used for the analysis of the information.

Results: Certain elements remained constant during the transition to maternity / paternity between generations, such as the practice of nurturing the promotion of good relationships between siblings. Dynamic aspects included taking care of physical and mental health, coercive control and school support. The constant practice in the first two generations and dynamic in the third, was to teach the role of girl or boy.

Conclusions: It is concluded that the transition to maternity / paternity is an internal process, while parenting practices depend on the sociocultural context.

Keywords: maternity, paternity, parenting practices.

\section{Resumo}

Objetivo: Analisar a transição para a maternidade / paternidade e a percepção das práticas parentais em três gerações de 18 homens e mulheres da cidade de Toluca, México.

Metodologia: Entrevistas em profundidade foram realizadas e o método fenomenológico foi utilizado para a análise das informações.

Resultados: Certos elementos permaneceram constantes durante a transição para a maternidade / paternidade entre gerações, como a prática de nutrir a promoção de boas relações entre irmãos. Os aspectos dinâmicos incluíam cuidar da saúde física e mental, controle coercitivo e apoio escolar. A prática constante nas duas primeiras gerações e dinâmica na terceira era ensinar o papel de menina ou menino.

Conclusões: Conclui-se que a transição para a maternidade / paternidade é um processo interno, enquanto as práticas parentais dependem do contexto sociocultural.

Palavras-chave: maternidade, paternidade, práticas parentais. 


\section{Introducción}

La familia a través de la historia ha sido considerada como una estructura presente en todo contexto sociocultural. Su relevancia radica en que tiene como función principal la socialización primaria, que para Berger y Luckmann (1968) implica que el sujeto se vaya constituyendo a sí mismo en un ser humano a través de la vida cotidiana. Así pues, el ser humano es parte de un sistema más vasto que lo socializa como medio de desarrollo, en él se encuentran los recursos sociales y la educación que son el modo en que una sociedad guía a un niño hacia las metas y las reglas que lo determinan (Bronfenbrenner, 1987).

En el proceso de socialización, se aprende la manera de ver la vida y como las personas se asumen a sí mismas y a los otros. La familia tiene diversas funciones como: brindar información sobre el mundo de acuerdo con un sistema de valores, enseñar códigos de conducta y ofrecer identidad (Musitu, Román y Gutiérrez, 1996).

La familia como ente universal responde a una necesidad humana básica que es la crianza, en la que ambos padres juegan un papel central (Malinowski, 1982). La función parental se constituye entonces como un reto que implica satisfacer las necesidades básicas de desarrollo de los hijos, tales como el cuidado físico y mental (Barudy y Dantagnan, 2009). Para lograrlo, los padres ponen en acción todo aquello que han aprendido ajustándolo a los nuevos contextos. En este sentido, Palacios y Rodrigo (2014) señalan que la familia es un escenario de convergencia intergeneracional, donde los adultos forman un puente hacia el pasado (la generación de los abuelos) y hacia el futuro (la generación de los hijos), siendo el afecto y los valores la vía de transporte entre las generaciones..

\section{La transición hacia la maternidad y paternidad}

El papel de los padres empieza desde el momento en que se da vida a un nuevo ser. Este hecho conlleva a cambios en la vida personal al ser responsable de otra vida e implica un nuevo rol con diferentes matices como ser educador, proveedor de afecto, de recursos materiales, apoyo, orientación, entre otros. Para Hidalgo (2014), el convertirse en madre o padre es una de las escasas experiencias en la vida del ser humano sumamente significativa y con alto impacto en la vida personal. Se caracteriza por distintos tipos de transición (satisfactoria, moderadamente satisfactoria, en solitario, tradicionales poco implicados) en los que se involucran además de la satisfacción, el apoyo social, disponibilidad de recursos, apoyo del cónyuge, valoración de la relación conyugal, reparto de tareas, ideas evolutivo-educativas, rasgos de personalidad y características sociodemográficas dominantes. 
Otros aspectos a destacar en esta transición son la elección de tener hijos o no, en la cual se involucran varios factores señalados por Deaton y Stone (2014) como: las normas sociales, la presión de los propios padres y las comunidades, o las contribuciones productivas de los niños y niñas. Para Puyana y Mosquera (2005), el significado del hijo primogénito se vincula con las relaciones entre hombres y mujeres y el ambiente sociocultural, ubicando tres tendencias: la tradicional, identificada por las disertaciones sobre la parentalidad de la década de los sesenta (madre dedicada al hogar, pilar del ambiente afectivo de la familia y padre proveedor y eje de autoridad); en transición, que se refiere los cambios socioculturales de un país, los encuentros culturales entre lo tradicional y moderno, las relaciones genéricas con ideas novedosas acerca del proveer, la autoridad y expresividad afectiva (participación del padre en la crianza, cuestionamiento del papel de proveedor y madres con multiplicidad de roles); y la tercera es la ruptura que implica "formas de ser padre y ser madre marcadamente diferentes a las tradicionales: las mujeres construyen proyectos de vida alternativos a la maternidad, mientras que los hombres se integran a las actividades hogareñas como proyecto de vida" (p. 6) (división de roles indistintos).

En cuanto a las expectativas, siempre se construyen con la llegada de los hijos, aún más con el primero, pues constituye un evento novedoso en la vida de todo ser humano, en palabras de Díaz, Pérez y Mozó (2009, p. 442), constituyen las "esperanzas, sueños, ilusiones de sus padres y madres hacia ellos".

Para Hidalgo (2014), existe una relación entre la historia vivida al convertirse en padres y el futuro desempeño como madre y padre. Sin embargo, en este eje temático de investigación, no se pretende partir de esta idea, sino más bien considerar el mundo subjetivo de los participantes, entendiendo que la transición a la maternidad/ paternidad conlleva aspectos que tienen que ver con lo emocional, con la naturaleza interna propia de cada persona y con sus experiencias cotidianas que no siempre son externalizadas y mucho menos a su propia descendencia.

Por otro lado, las prácticas de crianza se abordan desde la percepción como hijos, en el entendido que conlleva elementos objetivos cotidianos que permiten ver entre generaciones los cambios constantes y dinámicos.

\section{Las prácticas de crianza}

El estudio de las prácticas de crianza no es un tema nuevo, pero es preciso mencionar que a través de su estudio se ha delimitado su claridad conceptual, diferenciándose de otras definiciones como la actitud hacia la crianza o los estilos de socialización parental. Entonces, las prácticas son acciones concretas que llevan a cabo en la vida 
cotidiana las personas adultas (comúnmente madre y/o padre) encargadas de educar a los niños y niñas en un contexto específico. Para Oudhof y Robles (2014), implica el "qué" de la crianza y se enfoca en determinar las acciones que realizan los padres durante su socialización en el ámbito familiar, es decir, los actos concretos que llevan a cabo durante el proceso educativo.

Entre los antecedentes de este constructo, se encuentran la construcción de instrumentos de medición dirigidos a los padres, los cuales sentaron las bases para estudios posteriores con diferentes enfoques metodológicos y adaptaciones a contextos socioculturales diversos. En Estados Unidos de Norteamérica, Block (1965) estableció ocho prácticas: independencia, control, disfrutar con el niño, afecto negativo, expresión de afecto, énfasis de logro, guía razonada y castigo físico. En Holanda, Rink (2008) propone: comunicación mutua, cuidado de la salud física y mental, regularidad en las actividades cotidianas, recursos materiales suficientes, buena situación de ambiente y vivienda, relaciones en el hogar, buena red social, estimulación del desarrollo del hijo, límites y expectativas y control.

En México, los aportes sobre las prácticas de crianza también se remiten al terreno de la medición. En el sureste, Flores, Cortés y Góngora (2008) plantean: interacción positiva, disciplina positiva apoyo escolar, afecto positivo e intolerancia emocional. En el centro del país, Andrade y Betancourt (2008) proponen: comunicación/ control de conducta, autonomía, imposición, control psicológico, conducta prosocial, hiperactividad/falta de atención, síntomas emocionales y problemas de conducta. Por su parte, Rodríguez, Oudhof, González Arratia y Unikel (2011) desarrollan un instrumento en el que se mide el interés en las actividades, apoyo y orientación.

La crianza se ha considerado como un proceso bidireccional, en el que los hijos juegan también un papel protagónico. Estudiar la relación entre ambos contribuye a explicar y predecir la conducta de generaciones venideras (Merino y Arndt, 2004). Las bondades de la bidireccionalidad son destacadas por Vargas-Rubilar, Richaud y Oros (2018), con la implementación de un programa de fortalecimiento parental en Argentina.

En este sentido, considerando que las sociedades son dinámicas, es necesario cuestionarse si las prácticas de crianza idóneas para un contexto y tiempo específico pueden dejar de serlo de acuerdo a factores múltiples como las modas, las aportaciones científicas, la incorporación de creencias de otras culturas e incluso por las condiciones socioeconómicas cambiantes de un país o región. O por el contrario, si estas se mantienen constantes a través del tiempo en diferentes generaciones.

La pertinencia de estudiar estos elementos, en palabras de Rodrigo y Palacios (2014) implica que: "los padres co-construyen sus ideologías familiares, teorías 
implícitas y etnoteorías, ya construidas por otras generaciones, a partir de experiencias socioculturales directas (...) asociadas a la maternidad y la paternidad" (p. 289).

Asimismo, se relaciona con lo que Erikson (1983) Ilama generatividad, que consiste en la preocupación en la edad madura de guiar a las futuras generaciones y el mundo que se les deja, así como por la sociedad y el cuidado hacia los otros; esto es producto del encuentro de los miembros de la pareja conyugal y las generaciones que convergen en el núcleo familiar (Cigoli y Scabini, 2007).

El considerar a las sociedades en el tiempo como elementos dinámicos, brinda el justo motivo de continuar con la investigación en el campo de la crianza en la familia en diferentes generaciones. De igual forma, se precisa tener claridad sobre cuáles son los aspectos que permanecen en el tiempo. Si además se considera que México cuenta con una gran diversidad cultural, entonces, estos temas cobran aún mayor relevancia.

El objetivo de investigación fue analizar la transición a la maternidad/paternidad y la percepción de las prácticas de crianza en tres generaciones de hombres y mujeres.

\section{Método}

\section{Participantes}

En total, se trabajó con 18 hombres y mujeres, elegidos con base en los siguientes criterios; que contaran con tres generaciones consanguíneas en línea directa (3 abuelos, 3 hijos y 3 nietos; 3 abuelas, 3 hijas y 3 nietas). Buscar la homogeneidad en el total de los participantes no fue posible debido a la brecha generacional. Sin embargo, se consideraron algunos criterios generales en el momento del estudio relacionados con el aspecto socioeconómico como: tener su lugar de residencia en una zona urbana de la Ciudad de Toluca, Estado de México, no contar con problemas para la manutención diaria de la familia, acceso a los servicios de salud, disponer de todos los servicios (agua, luz, alumbrado público, pavimentación).

En cuanto a las diferencias por generación, se observó que en la primera (abuelos y abuelas) si bien habitaban en una zona urbana, originalmente su vida se desarrolló en una zona rural (lo cual es común en México con las personas de mayor edad), trasladándose a la ciudad por búsqueda de mejores oportunidades educativas y laborales para ellos o los hijos, o en su caso, para ser cuidados por éstos últimos. Por tal motivo, la segunda generación se caracteriza por un ir y venir del lugar de origen de sus padres (zona rural) a donde viven habitualmente (zona urbana). Este hecho ocurre 
en menor medida en la tercera generación, quienes en todos los casos son oriundos de la ciudad de Toluca y recuerdan vagamente el modo de vida de los orígenes de sus padres y abuelos.

Entre las características más resaltantes en las generaciones tanto en hombres como mujeres, se encuentran: aumento gradual en el nivel de escolaridad, la incorporación de la mujer al campo laboral y actividades mejor remuneradas en ambos sexos. En cuanto al número de hijos, se detecta menor descendencia (ver tabla 1).

Tabla 1. Características de los participantes de tres generaciones

\begin{tabular}{|c|c|c|c|c|c|c|}
\hline $\begin{array}{c}\text { Clave del } \\
\text { participante }\end{array}$ & Edad & Escolaridad & Ocupación & $\begin{array}{l}\text { Ocupación de } \\
\text { la pareja }\end{array}$ & $\begin{array}{l}\text { Años en } \\
\text { pareja }\end{array}$ & $\begin{array}{l}\text { Número } \\
\text { de hijos }\end{array}$ \\
\hline \multicolumn{7}{|c|}{ Mujeres } \\
\hline \multicolumn{7}{|c|}{ Primera generación } \\
\hline Abuela 1 & 70 & Secundaria & Hogar & Obrero & 50 & 4 \\
\hline Abuela 2 & 70 & Secundaria & Hogar & Fotógrafo & 52 & 5 \\
\hline Abuela 3 & 69 & Secundaria & Hogar & Pensionado & 50 & 4 \\
\hline \multicolumn{7}{|c|}{ Segunda generación } \\
\hline Hija 1 & 48 & Secundaria & Comerciante & Comerciante & 27 & 1 \\
\hline Hija 2 & 50 & Carrera técnica & Enfermera & Contador & 25 & 2 \\
\hline Hija 3 & 48 & $\begin{array}{l}\text { Lic. en } \\
\text { Educación }\end{array}$ & $\begin{array}{c}\text { Profesora de } \\
\text { primaria }\end{array}$ & $\begin{array}{c}\text { Ejecutivo de } \\
\text { ventas }\end{array}$ & 27 & 3 \\
\hline \multicolumn{7}{|c|}{ Tercera generación } \\
\hline Nieta 1 & 25 & $\begin{array}{l}\text { Lic. en } \\
\text { Educación }\end{array}$ & $\begin{array}{c}\text { Profesora de } \\
\text { primaria }\end{array}$ & Profesor & 4 & 2 \\
\hline Nieta 2 & 23 & Preparatoria & Vendedora & Desempleado & 1 & 1 \\
\hline Nieta 2 & 27 & Ingeniero & Empleada & Ingeniero & 2 & 2 \\
\hline \multicolumn{7}{|c|}{ Varones } \\
\hline \multicolumn{7}{|c|}{ Primera generación } \\
\hline Abuelo 1 & 86 & Primaria & Velador jubilado & Ama de casa & 54 & 6 \\
\hline Abuelo 2 & 74 & Secundaria & Cartero jubilado & Ama de casa & 50 & 5 \\
\hline Abuelo 3 & 70 & Secundaria & Obrero jubilado & Ama de casa & 48 & 4 \\
\hline \multicolumn{7}{|c|}{ Segunda generación } \\
\hline Hijo 1 & 51 & Abogado & Juez & Ama de casa & 30 & 4 \\
\hline Hijo 2 & 48 & $\begin{array}{l}\text { Maestría en } \\
\text { Educación }\end{array}$ & $\begin{array}{l}\text { Profesor de } \\
\text { preparatoria }\end{array}$ & $\begin{array}{c}\text { Profesora de } \\
\text { primaria }\end{array}$ & 25 & 1 \\
\hline Hijo 3 & 47 & $\begin{array}{l}\text { Ingeniero } \\
\text { mecánico }\end{array}$ & Empleado & Empleada & 22 & 2 \\
\hline
\end{tabular}




\begin{tabular}{|c|c|c|c|c|c|c|}
\hline $\begin{array}{c}\text { Clave del } \\
\text { participante }\end{array}$ & Edad & Escolaridad & Ocupación & $\begin{array}{l}\text { Ocupación de } \\
\text { la pareja }\end{array}$ & $\begin{array}{c}\text { Años en } \\
\text { pareja }\end{array}$ & $\begin{array}{l}\text { Número } \\
\text { de hijos }\end{array}$ \\
\hline \multicolumn{7}{|c|}{ Tercera generación } \\
\hline Nieto 1 & 29 & $\begin{array}{l}\text { Ingeniero en } \\
\text { sistemas }\end{array}$ & $\begin{array}{l}\text { Profesor de } \\
\text { preparatoria }\end{array}$ & Secretaria & 12 & 2 \\
\hline Nieto2 & 24 & $\begin{array}{l}\text { Lic. en Educa- } \\
\text { ción }\end{array}$ & $\begin{array}{c}\text { Profesor de } \\
\text { primaria }\end{array}$ & $\begin{array}{l}\text { Profesora de } \\
\text { secundaria }\end{array}$ & 4 & 1 \\
\hline Nieto 3 & 22 & Geógrafo & $\begin{array}{l}\text { Empleado de } \\
\text { gobierno }\end{array}$ & Desempleado & 1 & 1 \\
\hline
\end{tabular}

Fuente: elaboración propia

\section{Ejes temáticos y categorías}

En esta investigación se partió de dos ejes temáticos: Transición a la maternidad/ paternidad y Prácticas de crianza.

\section{Transición a la maternidad/paternidad.}

Considerando la transición a la maternidad/paternidad como un evento con características propias y únicas para quien lo vive, las categorías de análisis establecidas fueron:

- Elección de ser madre/padre: significado de la maternidad/paternidad y los motivos que llevaron a la elección de convertirse en madre/padre.

- Significado de los hijos: sentimientos y reacciones respecto a la llegada del primer hijo.

- Expectativas hacia los hijos: cambios significativos con el nacimiento y cómo fueron percibidos.

\section{Prácticas de crianza.}

El eje de prácticas de crianza permite analizar las actuaciones parentales que conllevan un carácter normativo sobre los aspectos que los padres deberían cuidar y atender durante la crianza (Oudhof y Robles, 2014). A partir de esto, las categorías iniciales fueron: cuidado físico y emocional, control, apoyo, relaciones en el núcleo familiar y enseñanzas recibidas. No obstante, durante el desarrollo de la investigación se modificaron de acuerdo con las aportaciones en el discurso de los participantes, quedando las siguientes: 
- Cuidado físico y emocional: el papel de la madre y del padre en el cuidado de los hijos, qué tanto estaban al tanto de sus necesidades físicas (aspectos materiales cotidianos: alimento, ropa, calzado, útiles escolares, cuidado de la salud física) y emocionales (brindar afecto, consuelo y responder a sus necesidades y demostraciones afectivas).

- Control coercitivo: control rígido, sin posibilidades de negociación y con efecto intimidatorio en los hijos.

- Apoyo escolar: acompañamiento para el logro de metas académicas.

- Relaciones entre hermanos: cómo era la comunicación, el trato entre los miembros y resolución de conflictos.

- Enseñar el papel de niño o niña. instrucción de los padres sobre cómo comportarse dentro y fuera del hogar de acuerdo con su sexo.

\section{Técnica de recolección}

Para aproximarse a la subjetividad de las y los participantes, se utilizó la entrevista a profundidad, con la finalidad de identificar los elementos constantes y dinámicos de la percepción de cada una de las generaciones de acuerdo con los ejes temáticos planteados (Transición a la paternidad y Prácticas de crianza). Si bien la idea central fue que los participantes se expresaran libremente, fue necesario realizar una guía de tópicos a partir de las categorías planteadas, las cuales permitieron ahondar en el tema sin desviarse del objeto de estudio. Para garantizar la claridad de la entrevista, se sometió a revisión por parte de tres investigadores expertos en el tema, quienes sugirieron cambios menores en la redacción de algunos tópicos que no alteraron su sentido.

\section{Procedimiento}

Con la finalidad de contar con las tres generaciones de hombres y mujeres pertenecientes al mismo núcleo familiar, se empleó la técnica de bola de nieve. En un primer momento se contactó a los primeros participantes, un hombre y mujer (padre y madre) que tuvieran con vida a sus progenitores, así como hijos de su mismo sexo. Por medio de su recomendación, fue posible tener con la participación de una segunda generación.

Cabe mencionar que contar con las tres generaciones de hombres y mujeres del mismo núcleo familiar no fue tarea fácil. Por esta razón, en un segundo momento, fueron los adultos de la primera generación quienes contribuyeron en mayor medida 
a contar con el total de los participantes, pues tenían mayor conocimiento sobre contemporáneos que tenían descendencia del mismo sexo.

Las entrevistas se realizaron de manera separada a cada miembro de la generación, con la finalidad de que las respuestas entre ellos no tuvieran elementos de presión o predisposición, tuvieran la libertad para expresarse y se evitara el conflicto familiar. Para ello, se acudió a la vivienda de cada uno de los participantes, con previo consentimiento informado, se les garantizó la confidencialidad de su identidad y el manejo de la información con fines académicos y de investigación. Se trabajó en dos sesiones, cada una con duración aproximada de cuatro horas.

\section{Manejo de la información}

Para manejar la información se seleccionó el método fenomenológico, el cual se consideró óptimo para esta investigación, ya que de acuerdo con Martínez (2004):

(...) respeta plenamente la relación que hace las personas de sus propias vivencias, ya que, al tratarse de algo estrictamente personal, no habría ninguna razón externa para pensar que ella no vivió, no sintió o no percibió las cosas como dice que lo hizo (p. 139).

El autor plantea siete pasos que permiten la comprensión del objeto de estudio y que son descritos para esta investigación de la siguiente manera. En primer lugar, se revisó el audio de cada una de las entrevistas de manera general para llegar a una primera comprensión (1). Se marcaron los enunciados de acuerdo con el discurso, con la finalidad de delimitar las categorías de acuerdo con los ejes temáticos (2).Con la finalidad de simplificar la información, se eliminaron las repeticiones y redundancias (3), lo que permitió aclarar y relacionar el significado de la categoría central; también se extrajeron las lexías representativas (4). Los hallazgos concretos se expresaron con un lenguaje científico, integrando los temas en una estructura particular descriptiva (5). Posteriormente, se realizó una estructura general, es decir, en una sola descripción para obtener lo que el autor llama fisonomía grupal, en la cual se identificaron aquellos elementos comunes que caracterizaron a los participantes de manera grupal de acuerdo con las categorías estudiadas (6). Finalmente, se dio a conocer de manera individual a cada uno de los participantes, los hallazgos encontrados en la investigación (7).

En el desarrollo del estudio, hubo una participación activa por parte de todos los participantes, incluso se observó en ellos un gran interés de expresarse respecto a la temática abordada, lo cual facilitó la obtención y análisis de la información. 


\section{Resultados}

Se presenta a continuación los resultados de los dos ejes temáticos con sus respectivas categorías.

\section{Transición a la maternidad/paternidad en hombres y mujeres}

\section{La elección ser madre/padre}

Los motivos para ser madre y padre son diferentes de acuerdo con el sexo de los participantes. Para las mujeres de todas las generaciones obedece a un "deber ser", sujeto a mandatos sociales, en los cuales la presión social de la pareja, la familia de origen y política y amistades jugó un papel fundamental. Si bien la tercera generación no dejó de ser caracterizada por estos elementos, agregaron que la decisión fue producto de tomarse el tiempo para reflexionar.

- Tuve hijos porque me lo exigían todos, mi familia en un principio me presionaba, también la familia de mi marido, mi esposo me decía que era 'mula', por eso me sometí a varios tratamientos para poder tener un bebé. (Hija 3).

- Me casé y quería ejercer mi carrera, de novios nunca hablé con mi esposo sobre tener o no tener hijos, pero cuando me casé, él luego luego quería hijos (...) lo pensé y dije, 'pues bueno', después me quise esperar otro poco y ya quería el otro, dejé de trabajar para cuidar a los niños porque nunca quiso que fueran a una estancia infantil. (Nieta 2).

Por su parte, los varones asumen la paternidad como algo "natural", que tiene que pasar en ellos por su condición de varón, como un deseo para perpetuarse, y en su caso como producto de la presión femenina.

- Tener hijos es algo normal, natural como hombre, sirven para formar una familia, aunque todas las noches me hagan enojar. (Hijo 3).

- Pues ella se embarazó y ni qué hacer tuve que 'juntarme' con ella. (Hijo 2).

\section{Significado de los hijos}

En el significado de los hijos no hubo diferencias entre generaciones, pero si por sexo, y fue en dos sentidos. Todos sin distinción, en un primer momento, lo asociaron a un 
sentimiento personal de realización, de felicidad por el nuevo ser que se ha engendrado, satisfacción e incluso bendiciones de un ser supremo.

- Para mí un hijo es todo es tu realización como mujer y como ser humano, la manera en que te proyectas y no son tuyos son como hijos de Dios que te los pone a tu cuidado para que le muestres el mundo y los cuides y les enseñes el amor a Dios. (Abuela 2).

- [...] un hijo es una bendición, mientras yo esté con ellos soy feliz. (Abuelo 2).

- Es motivo de gran satisfacción engendrar a un niño, un orgullo un privilegio hay muchos que quieren ser padres y no pueden y es muy bonito dejar que un niño te manipule. (Hijo 1).

En un segundo momento son las mujeres quienes presentan sentimientos de ambivalencia, pues encuentran al nuevo ser como precursor de nuevos retos para sostenerlo en sus necesidades diarias, motivo de esfuerzo extraordinario y posponer metas en los diferentes ámbitos como el trabajo, la vida profesional y cambios en los círculos sociales. Inclusive, surgieron atributos como: "son una carga", "trabajo duro" y "pérdida de libertad".

- Pues son bonitos los niños pero...pues si es duro cuidarlos y tenerlos. (Abuela 3).

- Para mí un hijo es una carga, solo tienes que trabajar para darle de comer, yo quería ser como doctora o maestra pero tenía que darle muy duro al trabajo para poder sacarlos adelante, no pude por ellos, por cuidarlos y atenderlos. (Hija 1).

\section{Expectativas hacia los hijos}

Las expectativas del hijo difieren principalmente entre hombres y mujeres. Las mujeres inicialmente esperaron del hijo que fuera un vínculo que fortaleciera la relación de pareja, que uniera y fuera motivo para que el cónyuge tuviera cambios positivos, dejar conductas nocivas como infidelidad, beber alcohol en exceso y agresividad. Posteriormente aparece la decepción del poder que se suponía tendría el hijo, y se observa como un elemento de crisis de pareja; incluso se vislumbra rivalidad, pues consideran que el nuevo integrante ha quitado la atención y cariño de la pareja.

Entre generaciones, los varones jóvenes aportan elementos más dinámicos que los de mayor edad. La connotación de las expectativas hacia el hijo en las dos 
primeras generaciones es visualizarlo como un miembro más que ayudará a trabajar, en la tercera, se encuentran centradas en aspectos emocionales y es considerado que será como un posible compañero, alguien en quien centrarse.

- Apenas nació el chamaco y llegaba, se asomaba para la cama y le hacía cosquillitas, a mí no, nada, yo pensaba 'todo por culpa del escuincle', toda su atención era para él. (Abuela 2).

- Desde chico he pensado que un hijo es un amiguito, un compañero que te acompaña...se comparten cosas de hombres...pero si es niña ni se diga, es la princesa de la casa, la consentida. (Nieto 1).

\section{Percepción de las prácticas de crianza}

\section{Cuidado físico y emocional de los hijos vs maltrato}

Es necesario precisar que esta categoría se centró inicialmente en el cuidado físico y emocional de los hijos. No obstante, de acuerdo con los hallazgos se añadió el maltrato. Los participantes de la primera y segunda generación de hombres y mujeres, narran las acciones de la madre, contrarias a lo que se entendería por cuidado y lucha por su supervivencia, enfatizaron la relación con sus padres como un evento significativo que marcó su vida por maltrato físico y emocional, frialdad afectiva, falta de comunicación, regaños frecuentes y sentimiento de rechazo. La tercera generación describe a una madre que cubría las necesidades de vestido, alimentación y afectivas.

Respecto al padre, en todas las generaciones predomina la percepción de figura ausente en cuanto a su presencia física y como figura afectiva. En las dos primeras es un proveedor parcial, poco cercano y quien ejerció menor maltrato físico. En la tercera generación se reconoce a un padre más pendiente y proveedor del hogar, también se reconoce una mayor convivencia, comunicación y confianza.

- Me golpearon muy duro, mi mamá me daba con un alambre de púas o con lo que encontraba hasta que mis abuelos papás de mis papás me recogieron cuando yo tenía poca edad. (Abuelo 1).

- Con mi mamá nunca me llevé bien, no fue una madre muy cariñosa, siempre era muy fría, nada cariñosa, me llevaba mejor con mi papá.(Hija 3). 


\section{Control coercitivo}

Un elemento constante que surge en todos los participantes es el control orientado hacia el castigo, considerado como un rasgo definitorio de ambos padres. Sin embargo, es a la madre a quien le atribuyen las dos primeras generaciones conductas de mayor crueldad. El control estaba centrado en las actividades del hogar (quehaceres domésticos), con poca o nula recreación, catalogándolo como excesivo, absoluto y caracterizado por la exigencia de la obediencia sin cuestionamientos, en caso de fallar el castigo físico fuerte era la opción.

De forma general, el control en la tercera generación se encaminaba hacia actividades cotidianas relacionadas con el orden y la manutención de la higiene de la vivienda, con los permisos para salir a jugar, realizar fuera de casa actividades escolares y las salidas con el grupo de pares. Específicamente, las mujeres consideraron que se vigilaban las relaciones de amistad o noviazgo con varones con la finalidad del cuidado del cuerpo respecto a la virginidad. Mientras que para los varones el control radicaba en evitar la mala conducta como salir sin permiso a divertirse, tardarse más de la cuenta del horario acordado o beber alcohol. De manera general, el castigo físico surge con menor severidad.

La madre, como autoridad mediadora entre los hijos y el padre, jugó un papel relevante, se perfiló como su portavoz respecto a lo que estaba o no permitido, dando cuentas al padre y en su caso, dejar a él la represalia.

- Éramos muchos hermanos, no se iban a estar ocupando de nosotros, pues no, cuando oíamos llegar a mi mamá, córrele a hacer el quehacer pendiente, porque se enojaba mucho si no teníamos todo listo, con la mano en la espalda nos daba duro. (Abuela 2).

- En unas cosas mi papá...y en otras mi mamá me tenía cortita, me colgaba del pelo si no estudiaba...pero el principal que supervisaba era mi papá. (Hijo 3).

\section{Apoyo escolar}

El apoyo escolar figura como una de las prácticas más relevantes para las generaciones, se encuentra de manera constante en el discurso. Absolutamente todos los que no lograron avanzar en el ámbito académico, añoran y lamentan el hecho.

Para las mujeres, el papel de la madre se posiciona como pilar para el logro o fracaso de metas académicas; las de la primera y segunda generación (las que consideran no haberlo logrado) son quienes las culpan, consideran injusta esta privación 
por las exigencias de la figura materna de dar apoyo doméstico y cuidado de los hermanos menores. Por el contrario, el discurso de los varones difiere, si bien consideran que existió la intención personal y de sus padres de educarse, el no haberlo logrado lo asumen como producto de causas externas a la familia, como el hecho de no contar con suficientes recursos económicos y la necesidad de su fuerza laboral que aportara ingresos en el hogar.

- $\quad$ Ni un grado, ni eso me dieron en mi casa, no porque me acuerdo que una vez traté de ir, sí fui un día a la escuela, y no, pero llegando, me llovió bien feo con mi mamá. (Abuela 1).

- Tengo mucho resentimiento con mi madre, nunca le importamos, yo quería estudiar, ser como doctora o aunque sea enfermera, ser alguien en la vida... no me dejó, no quiso teníamos que cuidar a los hermanos chiquitos... todavía no le perdono eso...me duele mucho. (Hija 3).

La tercera generación en general, considera que han logrado superar las expectativas académicas de sus padres, gracias al apoyo recibido para "ser alguien en la vida" y ser mejores personas. De igual forma el papel de las madres es crucial, con la búsqueda de recursos económicos extras, motivando, aconsejando, exigiendo y ayudando en las tareas escolares. Los que no lograron concluir la formación académica, lo atribuyeron a causas personales, manifestando frustración, tristeza y enojo y principalmente el sentimiento de haber fallado con la madre.

- Se me exigió muchísimo ser una buena estudiante, me ponía nerviosa cuando iba a llegar mi mamá...aunque me exigían sabía que era un bien para mí. (Hija 2).

- Mis papás me dieron la oportunidad yo era bien canijo quería andar de vago, con los amigos, las mujeres...mal, muy mal...me arrepiento mucho, mi mamá me decía 'ándale hijo estudia' 'échale ganas' para que te busques una novia buena que te ayude a trabajar bien y te cases... si no, no vas a encontrar más que a una mujer que le guste la calle... si estudias vas a tener mejor futuro. (Nieto 1).

\section{Relación entre hermanos}

El fomentar las buenas relaciones entre hermanos es una práctica constante que todas las generaciones vivenciaron por parte de sus padres, estas se centraron en 
el hecho de que por ser hermanos habría que: apoyarse mutuamente, respetarse, evitar pelea, realizar actividades en conjunto como, jugar, tareas domésticas y en su caso, académicas. Además, se tenían que visualizar como los mejores amigos para la vida. Asimismo, los hermanos figuraron como fuente de contención en las situaciones adversas.

- $\quad$ Siempre he querido mucho a mis hermanos nos decimos cosas pero ahí estamos, me enseñaron que son los mejores amigos que uno puede tener, en quien confiar y contarle los problemas... si fallábamos eran palizas a los dos, que porque le que le tocaba a uno le tocaba al otro. (Hija 1).

- Con tanto golpe, los momentos más felices eran cuando iba a cortar leña con mi hermano, jugamos y corríamos... si mi mamá nos miraba peleando, nos daba duro a los dos... siempre era que tenía que estar bien con ellos. (Abuela 1)

\section{Enseñar el papel de niño o niña}

El lenguaje y los simbolismos atribuidos a cada sexo, el "deber ser", "comportarse como hombre o mujer", se encontraron presentes en la infancia de cada una de los participantes.

En la primera y segunda generación, se tiene la claridad de que las prácticas se dirigieron a aspectos como: el uso de la vestimenta tradicional propia de cada sexo (mujeres: vestido, mandil; varones: pantalón, camisas), las actividades cotidianas del hogar; para las mujeres, aquellas relacionadas con el hogar para ser una buena esposa (lavar, planchar, cocinar, etc.), además del cuidado del cuerpo. En los varones actividades que requerían más fuerza física (cortar leña, cargar, reparar cosas del hogar). Asimismo, los participantes refieren que en la adolescencia existía un esfuerzo continuo por parte del padre para que se alejara de la madre con la idea de no adquirir conductas femeninas.

- Mi papá decía que nos debíamos dar a respetar con los muchachos y que las mujeres teníamos que llegar al matrimonio virgen...no andar ahí con varios hombres, no, que porque las mujeres solo valemos una vez nos decía mi papá, él nos cuidaba mucho y mi mamá igual, decía que si una sale bien la otra debe de salir igual, ninguna salió con su fracaso, nada. (Nieta 3).

- Primero seguía mucho a mi mamá para donde fuera, pero mi papá empezó a decir que estaba bajo sus faldas que tenía que hacer mis cosas solo 
y pues ni modo empecé a buscar a mi papá, aunque él siempre estaba trabajando...lo que aprendí de él fue que llegaba y ya pegaba un clavito, arreglaba una silla, ponía un foco y pues eso me gustó más que la plática de mujeres. (Hijol)

La tercera generación verbaliza las enseñanzas de manera más clara, marcando los juegos y juguetes atribuidos de manera tradicional para cada sexo (mujeres: muñecas, juegos de té, planchitas, escobas; varones: carros, muñecos, palas, carretillas) y el uso de la ropa tradicional. La manera de comportarse cotidianamente y la forma de expresarse y de comportarse con el sexo opuesto van de la mano (mujeres: no gritar, no pelear, no tomar iniciativa en aspectos de noviazgo, cuidado del cuerpo y la virginidad; varones; ser directos, arriesgarse, tener iniciativa con las mujeres).

Son las mujeres de la tercera generación quienes se revelaron en mayor medida a estas prácticas, sin embargo, optaron por llevarlas a cabo debido a la presión de los padres.

- [...] me comparaba con una prima, quería que fuera igual que ella, pero no, a mí me gustaban otras cosas, [...] ella quería que anduviera de vestidito, calcetitas y yo no quise nunca eso para mí. (Hija 2).

\section{Discusión}

\section{La transición a la maternidad/paternidad: Una constante entre generaciones}

En este primer eje de estudio que es la transición a la maternidad/paternidad, se reflejan aspectos constantes entre generaciones, con algunos tintes casi imperceptibles en los más jóvenes, que no hacen una diferencia contundente, ésta más bien se observó por sexo. Para las mujeres, el convertirse en madres se muestra como una elección obligada, mientras que en los varones es una cuestión natural propia de su naturaleza. En el significado de los hijos, todas las generaciones (sin importar el sexo) constituye una fuente de realización, de bendición, pero sabedores de la responsabilidad que implica; no obstante, en las mujeres hay ambigüedad, pues al mismo tiempo, el nuevo ser es una limitante en su desarrollo. En cuanto a las expectativas, ocurre algo similar; para ellas, son un motor que favorece el cambio positivo en la pareja y en la relación, pero se enfrentan posteriormente a una crisis de pareja; mientras que para ellos, los sentimientos positivos predominan. 
Estos hallazgos se explican desde una perspectiva más cultural, pues en México se asocia el concepto de familia con la existencia de niños en el hogar (Mendoza, 2006), principalmente cuando se pertenece a zonas en las cuáles los roles tradicionales del hombre y la mujer tienen mayor fuerza y en ella pesan más los mandatos sociales. Esto concuerda con Chodorow (1984), quien afirma que la asunción de la maternidad como una elección forzada, se relaciona primero con el aspecto biológico de lo femenino, en quien recae la función principal de dar vida; por otro lado, el aspecto sociocultural es el que determina en realidad sus elecciones.

De ahí que se pueda explicar la ambigüedad de sentimientos en las mujeres participantes hacia los hijos. Es decir, en una sociedad que sobrevalora la maternidad, postergarla tiene altos costos como la presión social constante, discriminación y prejuicios por parte de sectores como familia, pareja, amistades, compañeros(as) de trabajo e incluso la iglesia (Quintal, 2002). No así para los varones, en quienes la paternidad es la cúspide de la identidad masculina, plenitud y solidez (Parrini, 2000). Paradójicamente, en la transición, la implicación de los varones sigue siendo menor que en las mujeres a pesar de los avances en la participación igualitaria en las tareas domésticas y familiares (Serra, Gómez, Pérez-Blasco y Zacarés, 2014).

Por otro lado, es claro que la llegada del hijo genere sentimientos encontrados en los nuevos padres y madres, como se observó en la investigación de Puyana y Mosquera (2005) quienes encontraron que padres y madres de Bogotá, Colombia, refirieron el nacimiento del primer hijo como responsabilidad, en medio de la alegría y el temor ante el nuevo acontecimiento, relacionado con el responder a las necesidades económicas de los recién nacidos.

Pareciera que esto es común en contextos en los que predominan condiciones de pobreza. Deaton y Stone (2014) documentaron en un estudio con 1.8 millones de estadounidenses, que la evaluación de la vida y las experiencias hedónicas eran notablemente mejores en las parejas que vivían con un niño; pero al replicar sus hallazgos en regiones como África, América Latina, Oriente Medio y Asia del Sur, esto no fue así; pero sí, en países europeos ricos, cuyas características eran diferentes a otros sectores (más ricos, con mayor educación, más espirituales y más saludables). Contradictoriamente, encontraron que son precisamente los países pobres los que muestran más altas tasas de fecundidad, por lo que deducen que en estos lugares se elige tener hijos aun cuando disminuye el bienestar personal, pudiendo entonces existir otras razones como el no tener otra opción, o beneficios compensatorios relacionados con satisfacer los deseos de otros.

El tener altas expectativas hacia los neonatos y después observar otra realidad, tiene que ver con lo mencionado por Hidalgo (2014) e Hidalgo y Menéndez (2014), 
sobre la visión romántica de considerarlo motivo de fortalecimiento, estabilidad y cúspide de las relaciones de pareja; por el contrario, el nuevo miembro desencadena cambios, tensiones, redefiniciones de la propia vida y de las relaciones cercanas; incluso puede haber quebranto en la relación marital. Para Döring (2000) el pensar que "los niños y niñas unen" es totalmente falso, más bien, hacen más confusas las relaciones. Incluso para Flores-Ávila, Alejandre-Magaña y Martínez-Becerra (2016) afirman que cuando llega un bebé al hogar, se va complejizando su crianza y cuidados, por lo que la madres quien se agobia en mayor medida, ya que ella es la principal figura en ejercer estas funciones.

Los hallazgos de esta investigación, coinciden con las tendencias que Puyana y Mosquera (2005) proponen sobre la percepción de la llegada del primer hijo; se puede decir que la primera generación se ubica en la tradicional, la segunda en transición y la tercera se encuentra entre la de transición y la de ruptura, ya que aun no se observa de manera determinante que las mujeres sean proveedoras totales y que los hombres tengan como un proyecto de vida dedicarse al hogar, pero sí es claro que existe un involucramiento mayor de ambos en aspectos que tradicionalmente eran para un género.

Por otro lado, considerando la propuesta de Hidalgo (2014), en esta investigación la transición de las mujeres a la maternidad fue en solitario y moderadamente satisfactoria, mientras que para los varones fue más satisfactoria, pero con menor implicación.

Con la llegada de los hijos, mujer y hombre se convierten en madre y padre, lo que implica el ejercicio forzoso de la crianza que, de alguna manera, se relaciona con la perspectiva que se tiene sobre su propia madre y su padre. Este tema se trabaja en el siguiente apartado.

\section{Percepción de las prácticas de crianza: Elementos constantes y dinámicos entre generaciones}

El cuidado de la salud física y mental de los hijos, es una de las tareas primordiales de los padres; su opuesto es el abandono y el maltrato físico y emocional. De acuerdo con los hallazgos en esta investigación, la categoría inicial se modificó, quedando como "cuidado de la salud física y mental vs maltrato", debido a que este último, si bien se disipó en las dos últimas generaciones, predominó en los adultos de mayor edad con respecto a su madre. Ante esto, es preciso referir que las abuelas y abuelos vivieron en su infancia en condiciones de mayor marginación social y económica, hecho que cambió para los hijos y nietos al transitar la familia hacia la ciudad. Para 
Barudy y Dantagnan (2009) y Moreno (2004), es muy clara la relación entre el maltrato infantil y los contextos de injusticia social, pobreza y violencia; específicamente, las malas condiciones de la vivienda, el bajo nivel educativo de los padres y el no tener claridad acerca de las necesidades físicas y afectivas de los niños y niñas. Asimismo, el estrés experimentado por las madres por las condiciones de escasez pudieran ser también un detonador, que, de acuerdo con Ferreira, Lima, Vandenberghe y Celeno (2014), repercute y tiene un impacto negativo en la dinámica familiar y en el contexto social; además permite a las madres más proclives a ser violentas y/o negligentes.

Elementos similares se observaron en la práctica control coercitivo, caracterizada en la primera y segunda generación por castigo físico e imposición por parte de los padres. Para Posada-Díaz, Gómez-Ramírez, Ramírez-Gómez (2008), implica autoritarismo para cambiar comportamientos indeseables; es una forma de relacionarse con los hijos de manera impositiva, vertical y maltratadora.

Esta forma de control no es poco común en Latinoamérica. En México (Sauceda-García, Olivo-Gutiérrez, Gutiérrez y Maldonado-Durán, 2006), Argentina (Balzano, 2003) y Colombia (Pulido, Castro-Osorio, Peña y Ariza-Ramírez, 2013), muestran que padres y madres de familias que vivieron en épocas pasadas, tenían mayor predisposición al ejercicio del castigo físico hacia los hijos como principal medio de disciplina. Incluso, un estudio con familias españolas de Torres (2004), reveló que los varones con un promedio de edad de 46 años describían a sus padres como autoritarios, antiguos, intransigentes, sin posibilidad de dialogo, poco comunicativos y con carencias para mostrar sentimientos.

La tendencia a que los hijos repitan los mismos patrones es documentada por Pulido et al. (2013), en su estudio sobre la transmisión transgeneracional del castigo en familias nivel socioeconómico bajo de Bogotá, señalando que: "la mayoría de las pautas, creencias y prácticas fueron transmitidas en dos generaciones -la de los abuelos y abuelas y la de las madres y padres- (...)" (p. 257). Lo cual concuerda con los hallazgos de esta investigación.

Pulido et al. (2013) sugieren el estudio del castigo como práctica de crianza, sin categorizarlo como maltrato infantil. Block (1965) lo refirió como un factor en su instrumento Child Rearing Practices Report (CRPR), con él se pueden encontrar una diversidad de estudios a nivel mundial, lo cual indica su funcionalidad en diferentes contextos culturales. En México, Flores et al. (2008) lo incluyen en su instrumento para hacer referencia a la frecuencia con la que los padres regañan o llegan al castigo físico (pegar), cuando los hijos no cumplen con sus obligaciones.

Ante este panorama, es preciso reflexionar si el uso del control coercitivo con castigo físico de los padres es justificable de acuerdo al contexto sociocultural, o bien, 
si esta manera de corregir la conducta obedece más bien a una práctica que alude a una relación de poder en la cual, los adultos tienen un papel de supremacía con el que pueden llevar a cabo sin cuestionamientos esta práctica. De cualquier manera, no se puede perder de vista que la línea entre el control coercitivo con castigo físico y el maltrato infantil es muy delgada, y, por lo tanto, no es justificable bajo ninguna circunstancia o contexto.

Si bien en las nuevas generaciones fue referida con menor frecuencia, aún está presente; incluso, es preciso preguntarse, si esto es de la misma forma en zonas de mayor vulnerabilidad social en México. Lo ideal sería el ejercicio del control como una práctica de crianza que esté orientada a regular la conducta de los hijos por medio de un estilo parental menos autoritario y más democrático.

En cuanto al Apoyo escolar, se considera como una práctica dinámica, ya que a través de las generaciones, se notaron mayores logros académicos. Su presencia fue frecuente en el discurso de los participantes (ya sea por su presencia o por su ausencia), responsabilizando a la escasez económica y a la figura materna; esta última, es tradicionalmente la cuidadora principal y por lo tanto, es una fuente importante de apoyo y motivación para sus los logros. De manera general, la participación de la madre en actividades relacionadas con la escuela sigue siendo evidente, debido a que permanece más tiempo en el hogar o bien su actividad laboral es de tiempo parcial.

El impacto de la madre en este ámbito se refleja en algunos estudios. En Valencia, España, Martínez-Ferrer, Murgui-Pérez, Musitu-Ochoa, y Monreal-Gimeno (2008), señalan que el medio escolar es de gran relevancia en la vida del ser humano. En su trabajo con 1,068 adolescentes hombres y mujeres encontraron que un bajo apoyo de la madre se asocia con la minusvaloración de los estudios, mientras que un alto apoyo positivo se relaciona con la autoestima familiar y escolar.

Por otro lado, Solís-Cámara et al. (2007), en su estudio con 621 madres y sus hijos de cinco ciudades de México (Tuxtla Gutiérrez, Toluca, Cd. Victoria y Guadalajara), encontraron que los logros escolares están asociados con la escolaridad materna, así como con otros factores, como los ingresos familiares, el bienestar, expectativas y crianza. Esto último concuerda también con lo referido aquí por los participantes sobre la economía familiar como limitante para asistir a la escuela.

El apoyo escolar como una práctica de crianza específica no se encuentra suficientemente documentada en estudios mexicanos, a excepción de las investigaciones de Flores et al. (2008) en el Estado de Yucatán, quienes indagaron la percepción de niños y niñas sobre las prácticas de crianza, obteniendo que el apoyo escolar fue considerado por todos los participantes, pero tanto hombres como mujeres fueron enfáticos en el papel primordial de la madre. 
El papel de la madre en este aspecto se reflejó en las generaciones venideras, quienes lograron más y mejores resultados en el ámbito educativo; como señala Arancibia (1995), las acciones de la madre en la vida cotidiana contribuyen a que sus hijos se desarrollen cognitiva e intelectualmente para lograr un adecuado rendimiento y altos niveles de escolaridad y por lo tanto repetir este círculo. En este sentido, para Blanco y Umayahara (2004), en el siglo XXI, existe mayor preocupación en los padres por su futuro, por lo que se centran en su preparación académica y las actividades escolares (asistencia a la escuela, hacer tareas, estudio), así como en mejorar las condiciones de su educación. Para los autores, estas son actividades que se han incorporado al rol familiar, enriqueciendo así las pautas de crianza.

En cuanto a la Relación entre hermanos, desde la perspectiva de todos los participantes, ambos padres fomentaban relaciones mutuas positivas entre ellos. En México, el mantener unida a la familia es una de las funciones principales, y los hermanos son uno de los pilares. En esta categoría, no fue posible encontrar estudios exactos que documenten de manera concreta el cómo llevan a cabo esta práctica ambos padres en la vida cotidiana, pero una constante es que se fomenten la ayuda y apoyo en las actividades cotidianas y problemáticas, las manifestaciones de afecto, el diálogo, el reconocimiento a los logros y la convivencia. Por el contario, las acciones como pleitos, burlas, descalificaciones entre ellos se reprueban.

Una práctica similar a la referida aquí, es la propuesta por Rink (2008), en la que el educador debe cuidar que las relaciones mutuas y la comunicación en el hogar beneficien el desarrollo de los hijos. De manera específica, se refiere a la dinámica del grupo en el interior de la familia, la evitación de la formación de subgrupos, los papeles en el trato mutuo y el manejo de las normas, reglas y funciones. En este sentido, Ripoll, Carrillo y Castro (2009) señalan que una variable relevante en las relaciones padres e hijos y su influencia en las relaciones entre hermanos, es la percepción del tipo y la calidad del trato que reciben por parte de cada uno de sus padres. Cabe mencionar, que el estudio de la relación entre hermanos como una práctica podría sentar las bases para estudios posteriores debido a su escaso abordaje.

La última práctica, que es la enseñanza del papel de niño o niña, fue una constante en la vida de todos los participantes y de su socialización; tiene muy pocas variaciones entre generaciones, principalmente en la última, considerando que la competitividad y el logro fueron enseñados indistintamente entre hermanos.

Los papeles tradicionales dentro del hogar estuvieron presentes para cada sexo por medio de actividades concretas como: las tareas cotidianas (mujeres en actividades domésticas y varones fuera de la vivienda), la vestimenta, los juguetes asignados; en las mujeres el cuidado del cuerpo y el ser expresivas, en los varones 
ser fuertes física y emocionalmente. Estos hallazgos concuerdan con los de Torres, Ortega; Garrido y Reyes (2008) quienes al analizar la dinámica familiar con 203 padres y madres de familia del Área Metropolitana de la Ciudad de México, encontraron que: "(...) día a día se van haciendo las diferencias entre niños y niñas, en el trato diario, tipo de correcciones, enseñanzas, castigos, responsabilidades, tipo de juegos y juguetes" (p. 49). En consecuencia, los autores señalan que este trato diferenciado genera desigualdades de oportunidades, de libertades, de lenguaje y de expectativas, lo cual fue en efecto referido en esta investigación, principalmente por las mujeres más jóvenes.

La continuidad en las tres generaciones obedece al contexto sociocultural mexicano, pues como señalan Rocha y Díaz Loving (2012), este se ha caracterizado por un fuerte énfasis en la educación estereotipada, en donde hombres y mujeres aprenden a ser y a hacer, desde la infancia hasta la adolescencia.

Es preciso resaltar, que la generación más joven considera que si bien predominó la enseñanza de los roles tradicionales, también es posible visualizar cambios generacionales en mujeres y varones relacionados con los papeles de género atribuidos socioculturalmente, entre las que destacan, mayor participación en la crianza de los hijos por parte de los hombres, formas de relacionarse y de distribución de tareas más consensuadas y en las mujeres, mayor adquisición de niveles educativos e incorporación en el ámbito laboral. Igualmente, es posible observar que son más fuertes emocionalmente, competitivas y exitosas que en las generaciones pasadas.

Si bien los avances en materia de género han sido reportados ya en abundancia (Sarrió, Ramos y Candela, 2004; Estrada y Pérez, 2009: Chávez, 2015), es preciso considerar que no en todos los contextos se avanza de la misma forma. Por ejemplo, el lugar en el que crecieron las primeras generaciones fue rural, además de haber nacido y vivido en el siglo XX, situaciones que limitaron cambios sustanciales tanto en las prácticas de crianza como las relaciones de igualdad entre varones y mujeres. En lo que respecta a la educación de los hijos, Balzano (2003) señala que el papel de los contextos histórico sociales en los que se está inmerso tienen impacto para comprender cómo van surgiendo los nuevos referentes.

Entonces, los hallazgos de esta investigación dan cuenta del dinamismo de la cultura y cómo es posible lograr un cambio en los mandatos sociales atribuidos a varones y mujeres, lo cual permite relaciones más armónicas entre ambos y sientan bases para que generaciones venideras las fortalezcan y contribuyan a crear un mundo más justo.

De igual forma, existen datos novedosos en ambos ejes temáticos de esta investigación, pues se obtuvieron categorías aún poco exploradas en el estado del arte, como las contempladas en la transición a la maternidad/paternidad y en las 
prácticas de crianza (control coercitivo, relación entre hermanos y la enseñanza del papel de niño o niña). Entre las limitantes del estudio se encuentra que solo se trabajó en un contexto específico, motivo por el cual se sugiere continuar con el estudio de esta temática en ambientes que sean opuestos; por ejemplo, con personas en mayor vulnerabilidad social y económica y con aquellas que, por el contrario, tengan más ventajas económicas, sociales, educativas y culturales.

\section{Referencias}

Andrade, P. y Betancourt, D. (2008). Prácticas parentales: Una medición integral. En: S. RiveraAragón, R. Díaz-Loving, R. Sánchez-Aragón, y I. Reyes-Lagunes (Eds.), La Psicología social en México, Vol. XII. (pp. 561-565). Ciudad de México: Amepso.

Arancibia, V. (1995). El rol de la madre como mediadora en el proceso de habilitación. Estudios Públicos, 59, 251-264. Recuperado de https://www.cepchile.cl/cep/site/artic/20160303/asocfile/20160303184419/rev59_arancibia.pdf

Balzano, S. (2003). No todo el tiempo pasado fue mejor... percepciones de las diferencias generacionales en la crianza y educación de los hijos. Estudios sobre las Culturas Contemporáneas, IX(18), 103-126. Recuperado de http://www.redalyc.org/articulo.oa?id=31691807

Barudy, J. y Dantagnan, M. (2009). Los buenos tratos a la infancia. Parentalidad, apego y resiliencia. Barcelona: Gedisa.

Blanco, R. y Umayahara, M. (2004). Participación de las familias en la educación infantil latinoamericana. Recuperado de http://unesdoc.unesco.org/images/0013/001390/139030s.pdf

Berger, P. y Luckmann, T. (1968). La construcción social de la realidad: Buenos Aires: Amorrortu.

Block, J.H. (1965). The Child Rearing Practices Report (CRPR): A set of items for the description of parental socialization attitudes and values. Institute of Human Development: Berkley: University of California. Recuperado de http://faculty.tru.ca/wlroberts/block,1965.pdf

Bronfenbrenner, U. (1987). La ecología del desarrollo humano. Barcelona: Paidós

Chodorow, N. (1984). El ejercicio de la maternidad. Barcelona: Gedisa.

Chávez, J. C. (2015). Percepción de la igualdad de género en jóvenes universitarios. Revista de Trabajo social de la UNAM, 10, 75-90. 
Cigoli, V. y Scabini, E. (2007). Relación familiar. La perspectiva psicológica. En M. I. González (Coord.). El cuidado de los vínculos. Mediación familiar y comunitaria (pp. 71-108). Colombia: Universidad del Rosario. Recuperado de: https://books.google.com.mx/books ?hl=es\&lr=\&id=|GEAzg0XZogC\&oi=fnd\&pg=PA71\&dq=generatividad+familiar\&ots=r|yPQ6Xeib\&sig=1PH-Jdfew5ZGQE_gtfEIVta0vi4\#v=snippet\&q=fruto\%20de\%20un\%20 encuentro\&f=false

Deaton, A. y Stone A. (2014). Evaluative and hedonic wellbeing among those with and without children at home. Proceedings of the National Academy of Sciences of the United States of America PNAS, 11(4), 1328-1333. doi: 10.1073/pnas.1311600111.

Díaz, A., Pérez, M. V. y Mozó, P. (2009). Expectativas educacionales hacia hijas e hijos en una Escuela Rural de Alto Desempeño. Revista Interamericana de Psicología, 43(3), 442-448. Recuperado de http://pepsic.bvsalud.org/scielo.php?script=sci_arttext\&pid=\$0034-969020 09000300003\&lng=pt\&nrm=iso

Döring, M. T. (2000). La pareja o hasta que la muerte nos separe ¿un sueño imposible?, Ciudad de México: Fontamara.

Erikson, E. (1983). Infancia y sociedad. Buenos Aires: Horme-Paidós

Estrada, S. y Pérez, G. I. (2009). Actitudes hacia la igualdad de género: Comparación entre mujeres y hombres estudiantes de psicología e ingeniería. Archivos Hispanoamericanos de Sexología, XV(2), 67-95.

Ferreira, M., Lima, A. L., Vandenberghe, L. y Celeno, C. (2014). Estrés familiar en madres de niños y niñas y adolescentes con parálisis cerebral. Revista Latino-Americana de Enfermagen, 22(2), 440-447. Recuperado de http://www.scielo.br/pdf/rlae/v22n3/es_0104-1169-rlae-22-0300440.pdf

Flores-Ávila, L., Alejandre-Magaña A. B. y Martínez-Becerra E. (2016). Universidad pública y crianza de infantes. Reflexiones sobre su conciliación en la Universidad de Guadalajara. RA XIMHAI, 12(1), 83-104. Recuperado de http://www.redalyc.org/articulo.oa?id=46146696006

Flores, M.M., Cortés, M.L. y Góngora, E.A. (2008). Familia, crianza y personalidad: Una perspectiva etnopsicológica. Mérida: Universidad Autónoma de Yucatán.

Hidalgo, M. V. (2014). Transición a la maternidad y la paternidad. En J. Palacios \& M. Rodrigo (Eds.), Familia y Desarrollo Humano. (pp. 161-180). Madrid: Alianza Editorial. 
Hidalgo, M. V. y Menéndez, S. (2014). La pareja ante la llegada de los hijos e hijas. Evolución de la relación conyugal durante el proceso de convertirse en padre y madre. Journal for the Study of Education and Development, 26(4), 469-483. doi: http://dx.doi.org/ $10.1174 / 021037003322553851$

Malinowski, B. (1982). Estudios de psicología primitiva. Barcelona: Paidós Studio/Básica.

Martínez, M. (2004). Ciencia y arte en la metodología cualitativa. Ciudad de México: Trillas.

Martínez-Ferrer, B., Murgui-Pérez, S., Musitu-Ochoa, G. y Monreal-Gimeno. M. C. (2008). El rol del apoyo parental, las actitudes hacia la escuela y la autoestima en la violencia escoalr del adolescente. International Journal of Clinical and Health Psychology, 8(3), 679-692. Recuperado de http://www.redalyc.org/pdf/337/33712016004.pdf

Mendoza, Z. (2006). Saberes de mujeres y varones triquis respecto de la crianza de sus hijos: Cambios y continuidades generacionales. COLECTIVA, 2(1), 47-59. Recuperado de http:// www.scielo.org.ar/scielo.php?script=sci_arttext\&pid=S1851-82652006000100005

Merino, C. y Arndt, S. (2004). Análisis factorial confirmatorio de la Escala de Estilos de Crianza de Steinberg: validez preliminar de constructo. Revista de Psicología, XXII(2), 187-214. Recuperado de http://revistas.pucp.edu.pe/index.php/psicologia/article/view/6857

Moreno, J. M. (2004). Etiología del maltrato infantil: Estilo educativos prácticas de crianza y contexto social. Psicología y Salud, (001), 121-134. Recuperado de: http://www.redalyc.org/ pdf/291/29114113.pdf

Musitu, G., Román, J. \& Gutiérrez, M. (1996). Educación familiary socialización de los hijos. Barcelona: Idea Books S.A.

Oudhof, H. y Robles, E. (2014). Familia y crianza en México. Entre el cambio y la continuidad. Ciudad de México: Fontamara

Palacios, J. y Rodrigo, M. (2014). La familia como contexto de desarrollo humano. En M. J. Rodrigo \& J. Palacios (Eds.), Familia y Desarrollo Humano. (pp. 25-44). Madrid: Alianza Editorial.

Parrini, R. (2000). Los poderes del padre: paternidad y subjetividad masculina. En: J. Olavarría \& R. Parrini (Eds.), Masculinidad/es. Identidad, sexualidad y familia. (pp. 69-77). Ciudad de México: Flacso-Chile. 
Pulido, S., Castro-Osorio, J., Peña, M. y Ariza-Ramírez, D. P. (2013). Pautas, creencias y prácticas de crianza relacionadas con el castigo y su transmisión generacional. Revista Latinoamericana de Ciencias Sociales, Niñez y Juventud, 11(1), 245-259. Recuperado de http://revistaumanizales.cinde.org.co/rlcsnj/index.php/Revista-Latinoamericana/article/view/849/418

Posada-Díaz, A., Gómez-Ramírez, J. F. y Ramírez-Gómez, H. (2008). Crianza humanizada: una estrategia para prevenir el maltrato infantil. Acta Pediátrica de México, 29(5), 295-305. Recuperado de http://www.medigraphic.com/pdfs/actpedmex/apm-2008/apm085j.pdf

Puyana, Y. y Mosquera, C. P. (2005). Traer "hijos o hijas al mundo": significados culturales de la maternidad y la paternidad. Revista Latinoamericana de Ciencias Sociales, Niñez y Juventud, 3(2), 111-140. Recuperado de: http://www.scielo.org.co/scielo.php?pid=S1692-715X20050 00200005\&script=sci_arttext\&tlng=pt

Quintal, R. (2002). La presión social hacia las mujeres que desafían el paradigma "mujer igual a madre”. Revista GénEros, 9(27), 42-49. Recuperado de http://bvirtual.ucol.mx/descargables/548_presion_social.pdf

Rink, J.E. (2008). Pedagogía práctica en la situación familia. Toluca: Universidad Autónoma del Estado de México.

Ripoll, K., Carrillo, S. y Castro, J. A. (2009). Relación entre hermanos y ajuste psicológico en adolescentes: Los efectos de la calidad de la relación padres- hijos. Avances en Psicología Latinoamericana, 27(1), 125-142. Recuperado de http://www.redalyc.org/articulo.oaid= 79911627009

Rocha, T. E. y Díaz Loving, R. (2012). Identidades de género. Más allá de cuerpos y mitos. Ciudad de México: Trillas.

Rodrigo, M. J. y Palacios, J. (2014). Conceptos y dimensiones en el análisis evolutivo de la familia. En M. J. Rodrigo \& J. Palacios (Eds.), La familia como contexto de desarrollo humano. (pp.4589). Sevilla: EUROPA Artes Gráficas S.A. de C.V.

Rodríguez, B. Oudhof, H. González Arratia N. I. y Unikel, C. (2011). Desarrollo de una escala para medir la percepción de la crianza parental en jóvenes estudiantes mexicanos. Pensamiento Psicológico, 9(17), 9-20. Recuperado de http://www.redalyc.org/pdf/801/80122596001.pdf

Sarrió, M. Ramos, A. y Candela, C. (2004). Género, trabajo y poder. En E. Barberá \& I. Martínez (Coords.), Psicología y género. (pp. 193-216). Madrid: Pearson Prentice Hall. 
Sauceda-García, J. M., Olivo-Gutiérrez, N. A., Gutiérrez, J. y Maldonado-Durán, J. M. (2006). El castigo físico en la crianza de los hijos. Un estudio comparativo. Boletín Médico del Hospital Infantil de México, 6(3), 382-388. Recuperado de http://www.scielo.org.mx/scielo. php?script=sci_arttext\&pid=S1665-11462006000600004\&lng=es\&tlng=es.

Serra, E., Gómez, L., Pérez-Blasco, J. y Zacarés, J. J. (2014). Hacerse adulto en familia: una oportunidad para la madurez. En M. J. Rodrigo \& J. Palacios y (Eds.), Familia y desarrollo humano (pp. 141-160). Madrid: Alianza.

Solís-Cámara, P., Díaz, M., Del Carpio, P., Esquivel, E. Acosta, I. y De Jesús, A. (2007). La contribución del bienestar subjetivo, las expectativas y la crianza maternas en los logros escolares de sus niños y niñas y en la valoración de la participación de los padres. Acta Colombiana de Psicología, 10(2), 71-82. Recuperado de http://www.scielo.org.co/scielo. php?script=sci_arttext\&pid=S0123-91552007000200008\&lng=en\&nrm=iso\&tlng=es

Vargas-Rubilar, J. A., Richaud M. C. y Oros, L. B. (2018). Programa de promoción de la parentalidad positiva en la escuela. Un estudio preliminar en un contexto de vulnerabilidad social. Pensando Psicología, 14(23), 2382-3984. doi: https://doi.org/10.16925/pe.v14i23.2265

Torres, L. E. (2004). La paternidad: una mirada retrospectiva. Revista de Ciencias Sociales, 3(105), 47-58. Recuperado de: http://www.redalyc.org/html/153/15310504/

Torres L. E., Ortega, P., Garrido, A. y Reyes, A. G. (2008). Dinámica familiar en familias con hijos e hijas. Revista Intercontinental de Psicología y Educación, 10(2), 31-56. Recuperado http://www. redalyc.org/pdf/802/80212387003.pdf 